\title{
Burnout among hospital nurses in China
}

FRANCES LIN RN, BMN, MN (Hons) 1, WINSOME STJOHN P h D, R N, RM, MCHN, BAp p S c, GDi p E d, MNS 2 and CAROL McVEIGH PhD, RN, RM, MNS 3 1Lecturer, 2Associate Professor, Deputy Head of School, School of Nursing and Midwifery (Gold Coast), Griffith University, Queensland, Australia and ${ }_{3}$ Professor, Director of Nursing and Head of School, School of Health Sciences, Massey University Wellington, New Zealand

\section{ABSTRACT}

Aim: The aim of this study was to examine the level of burnout and factors that contribute to burnout in hospital nurses in the People's Republic of China.

Background: While burnout among hospital nurses has been widely researched in western countries, little research has investigated burnout among hospital nurses in China.

Method: A translated version of the Maslach Burnout Inventory-Human Services Survey was used to measure burnout in 249 randomly selected nurses from various wards of a large teaching hospital in Beijing, China. Questionnaire packs were sent to the hospital wards where selected nurses work. 128 nurses returned the completed questionnaire. The response rate was $51 \%$.

Results: showed moderate levels of Emotional Exhaustion and Personal Accomplishment, and low levels Depersonalisation. Age, years of experience, and professional title had a significant positive relationship with Emotional Exhaustion 
and Personal Accomplishment. Older, married nurses with more personal responsibilities and in a more senior position experienced higher levels of Emotional Exhaustion.

Conclusion: The findings suggest that burnout is a significant issue for nurses in China.

Implications for Nursing Management: The results of this study indicate that working environment factors such as relationships with co workers and managers may contribute to or mitigate burnout. There is a need to address personal and professional support, life-work balance, personal accomplishment and educational programs to reduce burnout in nurses working in China. 


\section{Introduction}

It has long been observed that nurses are at a high risk of burnout (Maslach 1982). To date, nursing burnout has been related to high workload, age, gender, education level, length of experience in nursing profession, and personal characteristics such as hardiness, coping strategies and social support (Cameron 1994, Maslach 1982). While burnout among hospital nurses has been widely researched in Western countries (Maslach \& Jackson 1996, Cameron 1994), little research has investigated burnout among hospital nurses in China, where a different culture and health system may result in different experiences for nurses.

In China, nursing leadership models and administrative strategies are different from those in Western countries. For example, in most Chinese public hospitals all nursing staff are permanent full-time and have to work 40 hours a week; there are no casual or on-call nurses for sick leave and holiday relief. Most hospitals still use a non-family friendly system with nurses rotating through a set roster. This type of rostering system offers limited choice for nurses to work around family life. Nursing is considered a lower-paid profession compared with other industries. As a result, nursing turnover and absenteeism can be high (Hu \& Liu 2004).

Social relationship patterns in China are also different from Western countries. Hierarchical social rules for parents and children, older and younger people, ruler and subjects are strictly observed in all aspects of Chinese people's social lives (Smith \& Tang 2004). For example, most people still look after their elderly parents at home. Sending parents to a nursing home is still seen as morally unacceptable. People are more conservative in expressing their opinions towards others. All of the above characteristics of the Chinese culture may play a significant role in nurses' burnout levels. Despite this, little research has investigated burnout in Chinese 
nurses. The extent and distribution of burnout in Chinese nurses is not known. Further, if it does occur, little is known about contributing factors among Chinese nurses.

This study examined burnout in hospital nurses in China and the relationship between social support (from family, friends, co-workers and managers) and socio-demographic factors, and the level of burnout among the nurses. Demographic factors examined were age, gender, marital status, qualification in nursing, length of experience in nursing, work position, professional title and clinical working areas.

\section{Background}

Maslach burnout theory (Maslach 1982) is been considered a seminal contribution to the field of burnout. "Burnout" describes the emotional reactions of staff whose work involves continual exposure to emotional and physical stresses, and it has been identified as a concern by nursing leaders (Maslach \& Jackson 1982). Burnout was defined as a "progressive deterioration in work and other performance resulting from increasing difficulties in coping with high and continuing levels of job related stress and professional frustration" (Paine 1984). Burnout has also been defined as human service workers' physical and emotional exhaustion where they gradually become indifferent towards their clients (Freudenberger 1980). According to Maslach:

Burnout is a syndrome of emotional exhaustion, depersonalisation and reduced personal accomplishment that can occur among individuals who do "people work" of some kind Maslach, 1982, p. 2).

Maslach and Jackson (1996) suggested that there were three core elements that comprise the burnout syndrome. The first component is Emotional Exhaustion, which results from having 
high intensity, long term contact with service recipients. The second component of burnout is Depersonalisation, which refers to showing a detachment beyond the requirements of professional distance from service recipients, seeing the recipients as objects and being indifferent when dealing with them. The third component, lowered Personal Accomplishment, is the feeling of low self-significance and self-worth that leads to inefficiency and possibly withdrawal from making an effort.

People who work in human service organizations consistently report lower levels of job satisfaction than people working in other types of organisations (Maslach \& Jackson 1996). In some cases human service professionals experience lower pay and longer hours. These industries are often more strictly regulated than others. Inadequate development opportunities for women and minority groups in lower status, lower paid positions are obvious in many health care areas (Tappen et al. 1998).

It has been reported that the risk of burnout is high in the nursing profession (Maslach 1982, Whitney and Ochaman, 1988). Nurse-patient interaction is focused on the patients' problems and the situations can be ambiguous and frustrating, because solutions for patients' problems may not be easily found. As Maslach and Jackson (1996) argued, for the person who works with people under these types of stressful situations for extended periods of time, burnout can occur.

Burnout among nurses is linked to job dissatisfaction, family problems and personal health issues (Song et al. 1997). Burnout is considered to be a leading contributor to serious problems in nurses' job performance and turnover (Song et al. 1997), with staff turnover a major reason for a worldwide nursing shortage (Cameron 1994, Persson 1993, Wright et al. 1993). Institutional costs of burnout include poor morale, poor quality of care, increased staff stress, and loss of management potential (Maslach \& Jackson 1996). 
Much of the stress experienced by nurses is related to the nature of their work: continued intensive, intimate contact with people who often have serious, sometimes fatal physical, mental, emotional and social problems. Furthermore, saving clients lives or helping them achieve a peaceful ending to their lives is not always successful. Despite nurses' best efforts, many clients get worse, not better. The continued loss of clients alone can lead to burnout (Tappen 1995). Even exposure to medical and antiseptic substances, unpleasant sights, and high noise levels can cause stress for some people. Health care providers experiencing burnout may become cynical or even hostile toward their co-workers and colleagues (Pape et al. 2005).

In high intensity clinical environments, however, Pines and Aronson (1988) reported that stress does not necessarily aways cause burnout. Burnout can be mitigated by personal characteristics such as hardiness, coping strategies, and social support (Berg 1994, Dick 1993, Duquette et al. 1995, Duxbury 1984, Wright et al. 1993). Maslach (1982) argued that, although burnout is avoidable and preventable, it takes effort from both the individual and the institutions to address it.

As discussed above, factors related to burnout are numerous, and are generally considered to have three dimensions: organizational factors, socio-demographic factors, and buffering factors. People are often able to succeed and be happy in stressful, demanding careers if they feel valued and appreciated and that their work has significance. They burn out when their work has no meaning and stress continuously outweighs support and rewards.

\section{Method}

The research sample was chosen from all full-time registered nurses working in a large public teaching hospital in Beijing. 


\section{Data collection instrument}

A translated Maslach Burnout Inventory-Human Services Survey-Chinese Version (MBIHSS-CV) (Pang et al. 1997) was used to examine the level of burnout among Chinese nurses. The survey tool is a twenty-two-item questionnaire with three subscales: nine items for Emotional Exhaustion (EE); five items for Depersonalisation (DP); and eight items for Personal Accomplishment (PA). The original English version of Maslach Burnout InventoryHuman Services Survey (MBI-HSS) has been widely tested and reported for internal consistency, test - retest reliability and validity (Maslach \& Jackson 1996). The reliability coefficients for the subscales reported by Maslach and Jackson (1996) were: 0.90 for EE, 0.79 for DP, and 0.71 for PA. Although the initial research using the MBI was carried out in the United States and Canada, subsequently it has been used for studies in many countries around the world, and it has been translated into various languages (Maslach \& Jackson 1996). The MBI-HSS was professionally translated into Chinese by Pang et al. (1997) in Hong Kong following questionnaire back-translation processes (English - Chinese - English). The reliability of the MBI-HSS-CV was reported as $\alpha=0.7737$ for Chinese nurses. I received permission to use the translated Maslach Burnout Inventory-Human Services Survey-Chinese Version (MBI-HSS-CV) from Pang (1997).

A researcher-developed demographic data sheet was used to collect data including gender, age, nursing qualification, years of working experience in nursing, position (RN, Head nurses, etc), clinical area of work, marital status. There are also three questions about the support they received from their co-workers, managers, family and friends, such as "do you receive enough support from your co-workers". Answers to these questions were "yes" or "no". No identifiable information was included in the demographic data sheet. 


\section{Sample and procedure}

This research was approved by the University and the Hospital's Ethics Committees. The target population was full-time Registered Nurses who provide direct care to patients in the in-patient setting from a large public teaching hospital (with more than 1000 beds) in Beijing, China. Among 935 full-time hospital nursing staff, a sample of 249 nurses was chosen with a stratified random sampling strategy from the hospital's human resources database according to specialties. Specialties of the sample consisted of the following: $30 \%$ of the nurses working in general medical, surgical, maternity and paediatric wards and all nurses working in Intensive Care Unit, Coronary Care Unit and Emergency department. All nurses in Intensive Care Unit, Coronary Care Unit and Emergency Department were selected because the total number of nurses in these clinical areas were small (7-12 nurses). The numbers in Intensive Care, Coronary Care and Emergency were small because only a small proportion of nurses working in these departments were permanent. Many nurses worked in these departments as part of a rotation program within the hospital. All male nurses were selected, because there were only a total of 3 male nurses working in this hospital.

Questionnaires were packed in Australia and sent to a research assistant in China. Each questionnaire pack included an information sheet for participants, a demographic data sheet and the Maslach Burnout Inventory-Human Services Survey-Chinese Version, and a return envelope labelled "human services survey". The packs labelled with participants' names were delivered to the participants' wards. Participants were asked to return the completed questionnaires in the envelope provided to a collection box located in the main reception of the hospital. All completed questionnaires were sent to the researcher in Australia. A total of 128 questionnaires were completed and returned which gave a response rate of $51 \%$. 
Participants' privacy and confidentiality were protected by giving them the choice to participate or not, and answering the questionnaires privately. Completion and return of the questionnaire was considered as consent to participate in the study. Participants were also assured via the information sheet that the responses on the questionnaires were anonymous, that the hospital would not be identified, and that no identifiable information would be published at any stage of the study.

\section{Data analysis}

The completed questionnaires were scored using the scoring key developed by Maslach and Jackson (1996). The final scores consisted of three subscores: Emotional Exhaustion (EE), Depersonalization (DP), and Personal Accomplishment (PA). Higher scores of EE, DP and a lower score of PA indicate a higher level of burnout.

Data were entered into SPSS 13.0 to carry out data analysis. Independent sample $t$ tests were applied to examine differences among groups for most of the variables such as age groups, gender, marital status, qualifications, working position, professional title in nursing, years of experience, and clinical working areas. The significance level used in this analysis was $\mathrm{P}<0.05$. One-Way ANOVA analysis, Kendall's tau-b correlation analysis were applied to examine the relationships among variables including emotional exhaustion, depersonalisation, personal accomplishment, age, years of experience, working position, qualification, and professional title in nursing. Descriptive statistics regarding the participants were also used in the data analysis. 


\section{Results}

Results in this study showed that the reliability coefficient for the total scale was $\alpha=0.75$, which was slightly lower compared with the result from the earlier study ( $\alpha=0.77$ ) (Pang et al. 1997) using the translated tool (Chinese version). The reliability coefficients for the three sub scales were: $\alpha=0.75$ for Emotional Exhaustion; $\alpha=0.66$ for Depersonalisation; and $\alpha=$ 0.72 for Personal Accomplishment.

The Burnout sub scores (Emotional Exhaustion, Depersonalisation and Personal Accomplishment) and their interrelationships with demographic data are displayed in Table 1. The Kendall's tau-b correlation matrix: relationships among variables are presented in Table 2. The total group burnout scores are showed in Table 3.

\section{Insert tables 1, 2 and 3 about here}

These results showed that the nurses' levels of Emotional Exhaustion and Personal Accomplishment were in the moderate level of burnout, while level of Depersonalisation indicated a low level of burnout according to Maslach's cut-off points scale (Maslach \& Jackson 1996). Correlation tests showed that level of Emotional Exhaustion and level of Depersonalisation had a significant positive relationship $(r=0.499)$, while level of Personal Accomplishment had a significant negative relationship with level of Emotional Exhaustion $(r$ $=-0.299)$ and level of Depersonalisation $(r=-0.258)$.

Age, years of experience, working position and professional title were significantly positively related to Emotional Exhaustion and were negatively related to Personal Accomplishment. 
Nurses who were married, senior and had higher professional titles experienced significantly higher levels of Emotional Exhaustion. Nurses aged 31-35 had the highest scores in Emotional Exhaustion was significantly higher than age group 21-25 $(p=0.001)$. Single nurses' Emotional Exhaustion score was significantly lower than the married group $(p=0.014)$. The Manager group's Emotional Exhaustion score was significantly higher than the nurse group ( $p=0.003)$. Nursing consultants' Emotional Exhaustion score was significantly higher than the other groups in the professional title category $(p=0.019$ and 0.007 respectively). Nurses working in ICU, CCU and emergency department experienced significant lower level of Personal Accomplishment than those nurses working in medical, surgical, obstetric and gynecological and pediatric wards $(p=0.003)$.

The $t$ test indicated that nurses who claimed that they received enough support from friends had significantly lower Depersonalisation scores while nurses who received enough support from coworkers and managers had significantly lower Emotional Exhaustion.

\section{Discussion}

\section{The Data Collection Instrument}

Results in this study showed that the reliability coefficient was slightly lower (0.75) compared with the result from the tool translator (Pang 1997) $(\alpha=0.77)$., More studies, such as test retest studies, need to be done to to develop a valid tool for use in the Chinese language .

\section{The Bumout Score}

The relationships among the scores of the three aspects of burnout (Emotional Exhaustion, Depersonalisation and Personal Accomplishment) found in this research were consistent with the findings of Maslach and Jackson (1996). Pearson's correlation test showed that Emotional Exhaustion had a significant positive relationship with Depersonalisation, while Personal 
Accomplishment had a significant negative relationship with Emotional Exhaustion and Depersonalisation. According to Maslach and Jackson (1996), the level of burnout is low if Emotional Exhaustion scores $\leq 16$, Depersonalisation scores $\leq 6$, and Personal accomplishment scores $\geq 39$. The level of burnout is moderate if Emotional Exhaustion scores 17-26, Depersonalisation scores 7-12, and Personal accomplishment scores 38-22. The level of burnout is high if Emotional Exhaustion scores $\geq 27$, Depersonalisation scores $\geq 13$, and Personal accomplishment scores $\leq 21$.

In relation to the total group, mean scores of Emotional Exhaustion and Personal Accomplishment indicated that this group of nurses experienced a moderate level of burnout, while Depersonalisation indicated that the group experienced a low level of burnout. The group scores present a positive view of the Chinese nurses in relation to Depersonalisation. This could be a result of social aspects of the Chinese culture. Embedded elaborate politeness rituals and protocols, and high expectations of social behavior reinforce the way Chinese people interact with each other (Chen et al. 2004). However, the results of Emotional Exhaustion and Personal Accomplishment may indicate that interventions are needed to address the burnout level of nurses in this environment.

The results of this study showed that younger nurses with less work experience, lower professional titles, and lower working positions experienced lower level of Emotional Exhaustion. This finding was supported by Foster (2003). However, this finding differs from Maslach and Jacksons's (1996), who found that older nurses in the United States experienced a lower level of burnout in Emotional Exhaustion, Depersonalisation, and reduced Personal Accomplishment than younger nurses. This was supported by Cameron's findings in Canadian nurses (Cameron 1994). The differences in the findings of these studies compared with this current study may be related to the era during which the studies were conducted, as 
nursing will have changed in the past 10 - 15 years. However another plausible explanation could be differences between the characteristics of the Chinese and Western society.

Many of the younger nurses who participated in this study fall into the first generation of the “One child per family” Chinese National Policy. Most of them, named as 'little emperors' when they were young by critics (Bakken 1993), may still be living with their parents by their late 20s. Because there is 'only one child', Parents and grandparents will usually take on most of the responsibility for daily living activities. Consequently, the 'only one child' generation may have fewer responsibilities in their lives than their counterparts in the past. This could be viewed as either advantage or disadvantage. Being well supported and not prone to burnout at the early age is an advantage, however, they could be more prone to burnout once they marry, have children and face more responsibilities later in life.

Another contributing factor may be that older women (especially married women) usually have more family responsibilities than younger women in China. Since the majority of the sample were female and married, the research findings regarding age and burnout may be related to social and cultural characteristics in China. In the past, men have been expected to financially support the family while the wives stayed at home to look after the family. A low income system in modern China requires dual incomes to keep families financially afloat. This means that women are doing everything that men do at work, yet they still have to cope with the roles and expectations of traditional Chinese culture - to be good around the household (Cooke 2007). In addition, due to the low wage system and a lack of established part-time arrangements for women in mainland China, a majority women work full-time (Cooke 2007). Further, working full-time for women is a status statement in the genderequality Chinese society. As a consequence, many Chinese women may feel greater stress 
when they get married, have children, and become older. These factors may explain why older nurses and married nurses experienced significant higher level of burnout.

The findings of this research showed that younger nurses experienced lower level of Personal Accomplishment. Younger Chinese nurses are at the beginning stages of their nursing career and, consequently, may not yet have achieved many of their career goals. These nurses may also work in lower positions. A lack of autonomy could lead to feelings of low personal accomplishment. Further, as explained in the Chinese proverb: 'juniors and seniors have their ranking', the power positions of individuals are fixed in the hierarchical Chinese society. Respect of seniors and obedience to authorities are considered virtues (Chan \& Chan 2005). It is expected that older people should be respected, regardless of whether they are correct or successful or not. Younger people are expected to respect and never challenge elders' opinions.

Another possible reason why older nurses with a greater experience and a higher professional title in nursing experienced higher level of personal accomplishment could be related to what they have achieved in their lives. They may have achieved a good position, a higher professional title as they gained experience, and they may have had a greater opportunity to start (or have started) a family. They may have earned the respect from younger nurses as they climbed up the ladder in the hierarchical Chinese social system (because they are older and senior). In another words, they may have met most of the expectations of the society for being successful. They may also have simply become wiser and have developed a more balanced perspective on their own personal accomplishments. However, no literature regarding marital status and working positions in other countries was found.

There were no significant differences found in level of burnout between male and female nurses. This finding was consistent with the findings of Kandolin (1993) and Wright et al. 
(1993). However, since there were only three male nurses out of 128 participants in this study, this finding can not be generalized. No significant differences were found in level of burnout between nurses with different levels of nursing qualification. This was consistent with Maslach's (1996) findings.

Results indicated that nurses working in the intensive care unit, coronary care unit and emergency department experienced lower levels of Personal Accomplishment. The possible reasons for this could be that these nurses face more death and dying. Another explanation is that they only care for patients for a short period of time when they are critically ill, because patients will be transferred to other wards when their clinical conditions become stable. Nurses working in an intensive care unit rarely see patients returning home when hospital treatments are successful. Being unable to see outcomes for patients they care for may lead to limited feelings of success.

There were no other significant findings across the clinical working areas. These results were consistent with most of the studies from other countries (Halm et al. 2005, Servellen 1993) which concluded that burnout appears to be much more influenced by factors related to the nature of nursing practice than by the area of clinical work.

Nurses who claimed to receive enough support from friends had a significantly lower level of Depersonalisation than the 'no' group, while nurses with support from coworkers and managers experienced significant lower levels of Emotional Exhaustion. These results were consistent with the findings of Bennett (1994b) and McAbee (1994), who found that social support and active coping were negatively related to burnout. These findings suggest that encouraging a positive working relationship among nurses and their managers may reduce the effect of stress from work and further prevent burnout. 
As the research sample was drawn from only one teaching hospital, generalisation of results is limited beyond the participating hospital. However other hospitals may be able to consider the applicability of these findings and make some interventions to prevent burnout in their nurses.

\section{Conclusions and Recommendations}

This study found that hospital nurses in China experienced higher levels of Emotional Exhaustion when they are older, married, worked in a higher position, and had a higher professional title. It appears that nurses with more responsibilities in their lives were more prone to Emotional Exhaustion, which is the core element of burnout. Nurses with greater support from coworkers, managers, and friends experienced lower levels of burnout, which may suggest that social support is a buffering factor to burnout.

There were no significant differences found in levels of Emotional Exhaustion and Depersonalisation across the various clinical working areas. However, nurses working in the intensive care unit, coronary care unit and emergency department experienced lower levels of Personal Accomplishment. This may suggest hospital decision-makers need to consider arranging for these nurses to work in other wards for periods of time during the year to buffer their negative experience at work.

There were some findings that were distinctively different from studies from Western countries. Younger nurses experienced lower level of Emotional Exhaustion than older nurses, which is different from most other studies. This may indicate that employers in Chinese hospitals should consider providing support services for older workers to assist them to cope with difficulties in their lives. Employers should also plan educational programs to encourage younger workers to be independent and learn to manage their own lives early. 
Burnout among nurses in China has different patterns of the distribution of burnout from nurses working in Western countries, which appear to be affected by cultural differences. 


\section{REFERENCES}

Bakken B. (1993) Prejudice and danger: The only-child in China. Childhood 1, 46-61.

Bennett L. (1994b) Quality of life in health care professionals: Burnout and its associated factors in HIV/AIDS related care. Psychology and Health 9, 272-283.

Berg A. (1994) Nurses' creativity, tedium and burnout during one year of clinical supervision and implementation of individually planned nursing care: Comparisons between a ward for severely demented patients and a similar control ward. Journal of Advanced Nursing 20, 742-749.

Cameron S.J. (1994) Job satisfaction, propensity to leave and burnout in RNS and RNAs: A multivariate perspective. Canadian Journal of Administration 7, 43-64.

Chan K.L. \& Chan C.L.W. (2005) Chinese culture, social work education and research. International Social Work 48, 381-389.

Chen X. He Y. \& Li D. (2004) Self-perceptions of social competence and self-worth in Chinese Children: Relations with social and school performance. Social Development 13, 570-589.

Cooke F.L. (2007) 'Husband's career first': renegotiating career and family commitment among migrant Chinese academic couples in Britain. Work, Employment and Society 21, 47-65.

Dick, M. (1993) Job burnout in RN to BSN students: Relationships to life stress, time commitments, and support for returning to school. Journal of Continual Education in Nursing 24, 105-109.

Duquette A. Kerouac S. Sandhu B.K., Ducharme F. \& Sai;moer P. (1995) Psychosocial determinants of burnout in geriatric nursing. International Journal of Nursing Studies 32, 443-456.

Duxbury M.L. (1984) Head nurses leadership style with staff nurse burnout and job satisfaction in neonatal care units. Nursing Research 33, 97-101.

Foster E.M. (2003) Stress, burnout and coping strategies of nurses practicing in two state public psychiatric hospitals in the New York metropolitan area,. Waldon University (Dissertation).

Freudenberger H.J. (1980) The high cost of high achievement, New York, Doubleday.

Halm M. Peterson M. Kandels M. Sabo J. Bullock M. Braden R. Gryczman A. Kroslp-Hagel K. Larson D. Lemay D., Sisler B. Strom L. \& Topham D. (2005) Hospital nurse staffing and patient mortality, emotional exhaustion and job dissatisfaction. Clinical Nurse Specialist 19, 241-251.

Hu, J. \& Liu H. (2004) Job satisfaction among nurses in China. Home Health Care Management and Practice 17, 9-13.

Kandolin I. (1993) Burnout of female and male nurses in a shift work. Economics 36, 141 147.

Maslach C. (1982) The cost of caring, Englewood Cliffs, New Jersy, Prentice-Hall Inc.

Maslach C. \& Jackson S.E. (1996) Maslach burnout inventory manual, Consulting Psychologists Press Inc.,Palo Alto CA. 
Mcabee R. (1994) Job stress and coping strategies among nurses. Association of Occupational-Health Nurses Standards Journal 42, 483-487.

Paine W.S. (1984) Professional burnout: Some major costs. Family and Community Health 6, $1-11$.

Pang M.C.S. Chin C.F. Choi K. Chung C.M. Ho L.M. Lam L. \& Leung A. (1997) Burnout in front line nurses in caring of the terminally ill, Hospital Authority, Hong Kong.

Pape T.M. Guerra D.M. Muzquiz M. Bryant J.B. Ingram M. Schranner B. \& Et Al. (2005) Innovative approaches to reducing nurses' distractions during medication administration. Journal of Continuing Education in Nursing 36, 108-116.

Persson L. (1993) Nurse turnover with special reference to factors relating to nursing itself. Scandinavian Journal of Caring Science 7, 29-36.

Pines A. \& Aronson E. (1988) Career burnout: Causes and cures, Collier Macmillan Publishers, London.

Servellen G.V. (1993) Burnout in hospital nurses: A comparison acquired immunodeficiency syndrome, oncology, general medical, and intensive care unit nurses samples. Journal of Professional Nursing 9, 69-77.

Smith, D.R. \& Tang S. (2004) Nursing in China: historical development, current issues and future challenges. International Journal of Nursing Studies 5, 16-20.

Song R., Daly B.J. Rudy, E.B., Douglas S. \& Dyer, M.A. (1997) Nurses' job satisfaction, absenteeism, and turnover after implementing a special care unit practice model. Research in Nursing \& Health 20, 443-452.

Tappen R.M. (1995) Nursing leadership and management: Concepts and practice, Davis company, Philadelphia.

Tappen R.M. Weiss S.A. \& Whitehead D.K. (1998) Essentials of nursing leadership and management, Davis company, Philadelphia.

Whitney P. \& Ochaman R.B. (1988) Psychology and productivity, Plenum Press, New York.

Wright T.F. Blache C F. Ralph J. \& Lutterman A. (1993) Hardiness, stress and burnout among intensive care nurses, Journal of Burn Care \& Rehabilitation 14, 376-381. 
Table 1: $\quad$ Emotional Exhaustion (EE), Depersonalisation (DP),

Personal Accomplishment (PA) scores of demographic variables $(n=128)$

\begin{tabular}{|c|c|c|c|c|c|c|c|c|}
\hline \multirow{3}{*}{$\begin{array}{c}\begin{array}{c}\text { Independent } \\
\text { variable }\end{array} \\
\text { Age }\end{array}$} & \multirow{3}{*}{$\begin{array}{c}\text { Group } \\
<20\end{array}$} & \multirow{3}{*}{$\begin{array}{r}\mathrm{N} \\
2\end{array}$} & \multicolumn{2}{|l|}{$\mathrm{EE}$} & \multicolumn{2}{|c|}{ DP } & \multicolumn{2}{|l|}{$\mathrm{PA}$} \\
\hline & & & \multirow{2}{*}{$\begin{aligned} \text { Mean } \\
34.0\end{aligned}$} & \multirow{2}{*}{$\begin{array}{r}\text { Std } \\
\text { \# }\end{array}$} & \multicolumn{2}{|c|}{ Mean Std } & Mean & Std \\
\hline & & & & & 1 & $\#$ & $\#$ & \# \\
\hline & $21-25$ & 46 & 18.3 & 9.5 & 4.0 & 4.7 & 33.3 & 9.3 \\
\hline & $26-30$ & 22 & 22.9 & 10.6 & 6.2 & 4.8 & 31.2 & 9.4 \\
\hline & $31-35$ & 27 & $* 28.3$ & 9.5 & 4.5 & 4.1 & 33.2 & 7.0 \\
\hline & $36-40$ & 12 & 25.4 & 10.6 & 2.6 & 2.5 & 35.3 & 9.1 \\
\hline & $41-45$ & 16 & 25.6 & 15.3 & 2.5 & 3.2 & 38.9 & 8.7 \\
\hline & $46-50$ & 1 & 21.0 & \# & \# & 15.0 & \# & \# \\
\hline & $51-55$ & 2 & 9.0 & \# & 3.0 & 3.2 & 45.0 & 4.2 \\
\hline \multirow[t]{2}{*}{ Gender } & Male & 3 & 18.3 & 9.0 & 4.0 & 1.7 & 29.7 & 2.5 \\
\hline & Female & 125 & 22.86 & 11.3 & 4.2 & 4.3 & 34.1 & 9.0 \\
\hline \multirow[t]{3}{*}{ Marital status } & Single & 46 & $* * 19.3$ & 9.4 & 3.5 & 3.7 & 33.9 & 8.7 \\
\hline & Married & 77 & 24.6 & 11.8 & 4.4 & 4.6 & 34.4 & 8.8 \\
\hline & Divorced & 2 & 31.5 & 9.1 & 7.0 & 1.4 & 22.5 & 2.1 \\
\hline \multirow[t]{2}{*}{ Qualification } & Certificate & 117 & 22.5 & 11.5 & 4.3 & 4.4 & 33.9 & 9.0 \\
\hline & Diploma & 10 & 24.7 & 7.9 & 2.7 & 3.3 & 34.7 & 8.5 \\
\hline \multirow[t]{3}{*}{ Experience } & Less than 10 years & 66 & 20.3 & 10.4 & 4.7 & 4.9 & $* * * * * * 32.8$ & 9.4 \\
\hline & $11-20$ years & 40 & $* 26.8$ & 9.8 & 3.9 & 3.5 & 34.3 & 7.6 \\
\hline & More than 20 years & 22 & 24.2 & 16.0 & 2.0 & 2.9 & 39.2 & 9.0 \\
\hline \multirow{2}{*}{$\begin{array}{l}\text { Working } \\
\text { position }\end{array}$} & Nurse & 37 & 20.7 & 10.3 & 4.4 & 4.7 & 33.0 & 9.1 \\
\hline & Manager & 90 & $* * * 27.5$ & 12.1 & 3.3 & 3.1 & 36.3 & 8.1 \\
\hline $\begin{array}{c}\text { Professional } \\
\text { title }\end{array}$ & Nurse & 57 & 21.2 & 10.2 & 4.8 & 4.6 & 32.4 & 9.5 \\
\hline
\end{tabular}




\begin{tabular}{|l|c|r|r|r|r|r|r|r|}
\hline & Nursing practitioner & 58 & 21.5 & 9.9 & 3.7 & 3.6 & 34.1 & 8.8 \\
\hline & Nursing consultant & 29 & $* * * * 28.7$ & 13.1 & 2.7 & 2.7 & 36.1 & 7.4 \\
\hline & Nursing specialist & 1 & 9.0 & $\#$ & 6.0 & $\#$ & 48.0 & $\#$ \\
\hline Working area & Medical ward & 30 & 23.3 & 9.2 & 3.5 & 3.1 & 34.5 & 7.6 \\
\hline & Surgical ward & 29 & 22.5 & 10.1 & 5.2 & 5.9 & 33.0 & 9.1 \\
\hline & Obstetric and gynec. & 16 & 22.0 & 11.6 & 4.5 & 4.3 & 35.7 & 8.8 \\
\hline & Paediatric ward & 14 & 24.2 & 15.6 & 3.6 & 4.0 & 34.5 & 7.1 \\
\hline & $\begin{array}{c}\text { Coronary critical } \\
\text { care }\end{array}$ & 10 & 21.1 & 10.4 & 2.5 & 1.9 & 33.7 & 7.4 \\
\hline & $\begin{array}{c}\text { Intensive care unit } \\
\end{array}$ & 12 & 21.5 & 12.1 & 5.3 & 4.9 & 32.2 & 10. \\
\hline & $\begin{array}{c}\text { Emergency } \\
\text { department }\end{array}$ & 7 & 22.7 & 11.2 & 4.1 & 4.3 & $* * * * 22.5$ & 8.9 \\
\hline
\end{tabular}

Note. Significance: $\mathrm{P}<0.05$ two tailed

\#The score was not available due to case number was one.

$*$ The score was significantly higher than age group $\underline{21-25}$ with $\underline{\mathrm{t}}=3.9, \underline{\mathrm{df}}=64, \mathrm{p}=.001$;

$* *$ The score is significantly lower than married group with $\underline{\mathrm{t}}=-2.5, \underline{\mathrm{df}}=110, \underline{\mathrm{p}}=.014$;

$* * *$ The score is significantly higher than nurse group with $\underline{\mathrm{t}}=3.0, \underline{\mathrm{df}}=113, \underline{\mathrm{p}}=.003$;

$* * * *$ The score is significantly higher than practitioner and nurse with $\underline{\mathrm{t}}=2.4, \underline{\mathrm{df}}=58, \mathrm{p}=$ .019 and $\underline{\mathrm{t}}=2.7, \underline{\mathrm{df}}=17, \underline{\mathrm{p}}=.007$ respectively;

$* * * * *$ The score is significantly lower than medical nurses with $\underline{\mathrm{t}}=-3.2, \underline{\mathrm{df}}=30, \underline{\mathrm{p}}=.003$.

****** The score is significantly lower than group "more than 20 years" with $\mathrm{t}=-1.1, \mathrm{df}=69$, $\mathrm{p}=0.029$. 
Table 2: $\quad$ Kendall's tau-b correlation matrix: relationships among variables $(\mathrm{n}=128)$

\begin{tabular}{lrrrrrrrr} 
& 1 & 2 & 3 & 4 & 5 & 6 & 7 & 8 \\
\hline 1. EE & 1.000 & & & & & & & \\
2. DP & $.499^{* *}$ & 1.000 & & & & & & \\
3. PA & $-.299^{* *}-.258^{* *}$ & 1.000 & & & & & \\
4. Age group & $.205^{*}$ & -.118 & $.237^{*}$ & 1.000 & & & & \\
5. Experience & $.228^{*}$ & -.108 & $.213^{*}$ & $.941^{* *}$ & 1.000 & & & \\
6. Working position & $.272^{* *}$ & -.120 & .168 & $.636^{* *}$ & $.644^{* *}$ & 1.000 & & \\
7. Qualification & .052 & -.101 & .027 & .096 & .080 & $.262^{* *}$ & 1.000 & \\
8. Professional title & $.197 *$ & $-.189 *$ & $.200^{*}$ & $.832 * *$ & $.836^{* *}$ & $.733^{* *}$ & .147 & 1.000 \\
\hline
\end{tabular}

** Correlation is significant at the 0.01 level (2-tailed)

* Correlation is significant at the 0.05 level (2-tailed) 
Table 3: $\quad$ Total group sub scores: Emotional exhaustion (EE), Depersonalisation (DP), Personal accomplishment (PA) $(\mathrm{n}=128)$

\begin{tabular}{|l|c|c|c|c|}
\hline \multicolumn{1}{|c|}{$\begin{array}{c}\text { Dependent } \\
\text { variables }\end{array}$} & Minimum & Maximum & Mean & Std. Deviation \\
\hline EE & 1.00 & 70.00 & 22.75 & 11.25 \\
\hline DP & 0.00 & 23.00 & 4.17 & 4.34 \\
\hline PA & 0.00 & 39.00 & 33.90 & 8.90 \\
\hline
\end{tabular}

Note: Maslach \& Jackson's MBI Sub scores (1996)

Rang of experience of burnout:

EE: Low: $\leq 16$ Average: $17-26$

High: $\geq 27$

DP: Low: $\leq 6$ Average: 7-12

High: $\geq 13$

PA: Low: $\geq 39$ Average: $38-32$

High: $\leq 31$ 